\title{
LA URGENCIA DE LOS DERECHOS: EXIGIBILIDAD EN EL MARCO DE LA DESIGUALDAD DE GÉNERO
}

LAURA PAUTASSI ${ }^{1}$

UNIVERSIDAD DE BUENOS AIRES

ARGENTINA

"He soñado con la idea de una democracia y una sociedad libre en la cual las personas viven juntas en armonía y con igualdad de oportunidades. Es un ideal el cual quiero vivir para verlo hecho realidad. Pero si para ello es necesario... es un ideal por el cual estoy preparado a morir". 


\title{
RESUMEN
}

l artículo se sitúa en el marco de los acuerdos alcanzados en pactos y tratados in-
ternacionales y en el reconocimiento del cuidado como derecho y las consiguientes
obligaciones positivas y negativas que implica. Para ello analiza la base de la injus-
ta división sexual del trabajo, sea remunerado o no, y la necesaria revisión de esta

Palabras Claves: enfoque de derechos, cuidado, trabajo, género.

\begin{abstract}
This article comes in the frame of agreements reached in international pacts and treaties and in the recognition of care as a right, and in the resulting positiv and negative obligations. For that the article analyses the base of the unjust sexual division of labor, independent if it is paid or not, and the necessary revision of this situation of extreme unfairness looking ahead to the regional agenda post 2015, where the urgency of treating these situations, in the frame of political, economical, social, familiar and demographic transformations, imposes public policies that are respectful of the human rights.
\end{abstract}

Key words: rights aproach, care, work, gender.

\section{LOS DERECHOS EN LA AGENDA PÚBLICA}

Es indiscutible la importancia que ha tenido la incorporación de una agenda de derechos humanos a nivel de la políticas públicas en América Latina en los albores del siglo XXI, donde la inclusión de políticas de igualdad de género ha ocupado un lugar destacado, particularmente a la luz de las demandas y esfuerzos que los diversos movimientos de mujeres y de derechos humanos han presionado para su incorporación. Es que en este continente, el que es considerado el más desigual de la tierra, las demandas y urgencia por la efectividad de los derechos se conjugan al mismo tiempo que las presiones económicas, políticas, familiares y demográficas confluyen en una necesidad insoslayable de revisar la matriz de lo "público" y donde la provisión de bienes y servicios sociales, en especial los vinculados con el cuidado, cobran centralidad no solo como una demanda de justicia sino como requisito indispensable para promover

\footnotetext{
${ }^{1}$ Investigadora independiente, Consejo Nacional de Investigaciones Científicas y Técnicas (CONICET) y la Facultad de Derecho, Universidad de Buenos Aires, Argentina. Una primera versión de este trabajo fue presentando en el marco del 58으 período de sesiones Comisión de la Condición Jurídica y Social de a Mujer (CWS) Naciones Unidas, Nueva York, 10 al 21 de marzo de 2014 , Evento Paralelo "Logros y desafíos en la implementación de la agenda de género en los ODMs y en la Agenda de Desarrollo post 2015"-
} 
el desarrollo. Entre otras razones, porque este modelo de provisión del bienestar asentado en la falta de reconocimiento del valor del trabajo no remunerado, prestado principalmente por mujeres, no solo da cuenta de una injusta y desigual distribución de las obligaciones, sino que ha mostrado límites a la hora de promover sociedades más igualitarias.

Es precisamente bajo este escenario, que resulta necesario recuperar el significativo aporte que tuvo la Declaración del Milenio² $^{2}$ a partir de la cual se introduce formalmente el denominado "enfoque de derechos", y se instala como una metodología que persigue un objetivo ético político como es la equidad social y de género. Así, el enfoque de derechos utiliza el marco conceptual que brindan los derechos humanos para aplicarlos a las políticas públicas y al desarrollo en su conjunto, a partir de considerar los principios, reglas y estándares que componen el derecho internacional de los derechos humanos, que ha desarrollado un corpus que establece con mayor exactitud no sólo las obligaciones negativas del Estado sino también un conjunto de obligaciones positivas. Implica, entre otros efectos, que se ha precisado no sólo aquello que el Estado debe abstenerse, a fin de evitar violaciones a derechos y garantías ciudadanas, sino también aquello que debe hacer en orden a lograr la plena realización de los derechos civiles, políticos y también económicos, sociales y culturales (DESC).

En consecuencia, se ha conformado un marco conceptual, pero con componentes teóricooperativos, en donde los estándares fijados por el Sistema Internacional de derechos Humanos, como en los sistemas regionales -en nuestro caso el Sistema Interamericano de Protección de Derechos Humanos (SIDH) - en temas tales como universalidad, progresividad y no regresividad, el derecho a la igualdad, el derecho de acceder a la justicia y el derecho a la participación política, ocupan un lugar central al momento de fijar pautas y criterios para el diseño e implementación de estrategias de desarrollo (Abramovich y Pautassi, 2009). Implica pues, transformar el accionar del Estado, y no solamente revisar ciertas dinámicas de distribución de satisfactores, ya que para que cada intervención estatal sea respetuosa de los derechos civiles, políticos (DCP) y económicos, sociales y culturales (DESC), debe incorporar estándares de derechos en la definición de políticas y estrategias de intervención tanto de los Estados como de los actores sociales y políticos locales, al igual que los organismos de cooperación para el desarrollo, como también para el diseño de acciones para la fiscalización y evaluación de políticas sociales.

Pues bien, sobre el contenido de estos derechos, el Sistema Universal (SU) y el Interamericano de Derechos Humanos (SIDH) han realizado aportes fundamentales, especialmente a partir de los desarrollos que ha realizado el Comité de Derechos Económicos, Sociales y Culturales del Pacto Internacional de Derechos Económicos, Sociales y Culturales (PIDESC) como también el Comité para la Eliminación de la Discriminación contra la Mujer (Comité CEDAW). Con igual fuerza imperativa, los órganos competentes del Sistema Interamericano de Derechos Huma-

\footnotetext{
${ }^{2}$ Aprobada por la Asamblea de Naciones Unidas en el año 2000, donde los Estados no solo reafirmaron sus compromisos para la protección y efectivización de derechos humanos, sino que por primera vez se acordaron ocho objetivos de desarrollo que deben ser medidos a partir de un plazo establecido (año 2015). Al respecto, ver entre otros, Oficina del alto comisionado de Naciones Unidas para Derechos Humanos, www.ohchr.org/
} 
nos (OEA), como el Protocolo Facultativo de la Convención Americana de Derechos Humanos en materia de DESC, al que conocemos como "Protocolo de San Salvador", que al igual que la Convención Interamericana para Prevenir, Sancionar y Erradicar la Violencia contra la Mujer, conocida como Convención de Belém do Pará, suman la labor de los órganos de vigilancia específicos, Comisión Interamericana de Derechos Humanos y la Corte Interamericana de Derechos Humanos, las relatorías especiales que han otorgado el corpus regional a los mandatos estatales y un reaseguro a los sujetos titulares de derechos del continente. El denominado contenido mínimo de un derecho, el principio de aplicación de progresividad y no regresividad en los derechos, garantías de acceso a la justicia, en sentido amplio, y a la información pública constituyen estándares que deben ser cumplidos por los Estados. Es decir, están jurídicamente obligados a hacerlo por haber ratificado los citados instrumentos de derechos humanos ${ }^{3}$.

Estos estándares a su vez han permitido el desarrollo de indicadores de derechos humanos, esto es, parámetros o unidades de medida de tipo cuantitativo que pueden lograrse y verificarse en relación con un criterio, que en este caso son los estándares. A su vez, en derechos humanos es importante que se complemente la verificación empírica con técnicas cualitativas, como la utilización de las señales de progreso cualitativas, las cuales se caracterizan porque no parten de una categoría preestablecida, como tampoco de una escala de medición ya dada (estadística), sino que captan la definición de la situación que efectúa el propio actor social y el significado que este le da al fenómeno evaluado, que resultan claves para poder interpretar los hechos. En consecuencia, la opción consiste en definir indicadores cuantitativos y señales de progreso cualitativas para verificar el grado de cumplimiento de la obligación contenida en el derecho. En el caso del SIDH se ha adoptado el sistema de indicadores de progreso para medir los esfuerzos de los Estados en los informes nacionales previstos en el Protocolo de San Salvador ${ }^{4}$, y en la misma dirección, los Estados deben elaborar sus informes de país vinculados a los esfuerzos por garantizar una vida libre de violencia contra las mujeres en el marco del proceso de monitoreo que desarrolla el Mecanismo de seguimiento de la Convención de Belem do Pará5.

En rigor, el enfoque no refiere a un conjunto de prestaciones en los diferentes campos de actuación de la política social (salud, vivienda, educación, entre otros) sino que esencialmente se trata de una obligación mínima pero ineludible, de contenido universal, que apunta a asegurar la satisfacción de por lo menos niveles básicos de cada uno de los derechos sociales e incluye obligaciones de comportamiento y obligaciones de resultado pero siempre con el mandato de alcanzar la satisfacción plena de cada derecho. En particular, si bien en todos los Pactos se contempla una realización paulatina del contenido de derechos, considerando especialmente las restricciones derivadas de la limitación de los recursos con que cuentan los

\footnotetext{
${ }^{3}$ Tal es el caso de Argentina, que con la reforma constitucional de 1994 incorporó con jerarquía constitucional 11 tratados de Derechos Humanos, entre los que se incluye la CEDAW y en el año 2007 ratificó el Protocolo Facultativo, del mismo modo que en el 2011 ratificó el Protocolo Adicional del PIDESC, www2.ohchr.org/english/

${ }^{4}$ Para mayor información, visitar: www.oas.org/es/sedi/ddse/paginas/index-7_GT.asp

${ }^{5}$ Se trata del Sistema de indicadores de progreso para la medición de la implementación de la Convención de Belém do Pará, www.oas.org/es/mesecvi/proceso.asp
} 
Estados, al mismo tiempo impone varias obligaciones con efecto inmediato y que se relacionan con el estándar conexo de la utilización del máximo de recursos disponibles.

En otros términos, el enfoque de derechos habilita una interpelación directa a la forma en que se han venido implementando las respuestas estatales en el campo económico, social, político y cultural, aportando herramientas de análisis e intervención sobre la "estatalidad" de modo de transformar la lógica, las formas y el contenido de dichas respuestas. Al respecto, surgen numerosos interrogantes que se vinculan con el "nudo" central que, entre otras razones, esta dificultando la aplicación de políticas públicas respetuosas del conjunto de derechos humanos ${ }^{6}$.

En los hechos, y a pesar de los importantes avances que los Estados han realizado en este joven siglo XXI, queda aun un extenso camino por recorrer en materia de satisfacción de las obligaciones positivas de los Estados en materia de derechos humanos, en especial para las mujeres. Volviendo al inicio, la agenda post 2015 aparece cargada de demandas legítimas para lograr un efectivo avance en la concreción de los Objetivos de Desarrollo del Milenio (ODM), tanto a partir de discutir la inclusión de nuevas definiciones o aquellos "objetivos olvidados" como también por la necesidad de avanzar en el efectivo goce de los derechos. Al respecto, si bien ya se han identificado numerosos objetivos para debatir e incluir en la agenda global, surge con fuerza la necesidad de considerar como nuevo ODM a la redistribución, entendida la misma en su vertiente económica, social, simbólica, cultural pero especialmente transformadora de las inequidades presentes ${ }^{7}$. Al respecto, pensar en cómo redistribuir el acceso a bienes y servicios, al tiempo, a la cultura, a los derechos, al tiempo libre, al bienestar económico, a los puestos de conducción, a la toma de decisiones privadas y públicas, a las garantías para una vida libre de violencia para las mujeres, al cuidado, tanto para recibirlo como para brindarlo.

Lo expuesto remite nuevamente a los temas invisibilizados durante años y años, como lo es el cuidado (otrora denominado trabajo reproductivo y actualmente economía del cuidado) y quien lo ejerce (para sí o para otros) dejando en claro un problema de ejercicio de derechos, cuando éstos están acordados, o en caso contrario, de disminución de desigualdades, como condición de una política pública (Pautassi, 2007a). En uno y otro caso, se debe enfatizar que es necesario abrir un debate en donde se entrecruzan derechos, desigualdades y política, en consonancia con las clásicas demandas feministas y sus desarrollos teóricos, con la especificidad que, si en la situación actual no se amplía el ejercicio de estos derechos, se seguirá afectando el principio de igualdad, principio fundamental para el desarrollo de la persona y por ende de las sociedades. Estos son los aspectos que ocupan el interés del presente artículo.

\footnotetext{
$\overline{{ }^{6} \text { En Pautassi y Gamallo (2012) y Pautassi (2014) se presentan resultados de investigaciones que han indagado sobre la incorpora- }}$ ción del enfoque de derechos en la Argentina de la posconvertibilidad.

${ }^{7}$ Cabe recordar una vez más los valiosos aportes de Nancy Fraser (2000) con el dilema de la redistribución y reconocimiento, que en el caso latinoamericano cobran una dramática actualidad.
} 


\section{DE LA INVISIBILIDAD A SU NECESARIA INCLUSIÓN: EL CUIDADO SIEMPRE PRESENTE}

La relevancia actual de la problemática del cuidado ${ }^{8}$ y las dimensiones actuales que ha adquirido en América Latina da cuenta del agotamiento de múltiples situaciones de desigualdad. En primer lugar, la división sexual del trabajo, como expresión de las relaciones de género en el mundo del trabajo, que ha estructurado y consolidado las relaciones de subordinación de las mujeres, cuya manifestación es su concentración en las responsabilidades de cuidado, y su menor y desigual participación en el mercado laboral (Pautassi, 2007b; Rodríguez Enríquez, 2007b).

Considerando que la división sexual del trabajo está en la base de la desigualdad de las relaciones sociales de género, es necesario analizar las múltiples manifestaciones que se expresan en la menor posibilidad de las mujeres para incorporarse al mercado de trabajo remunerado, para ejercer sus derechos, resolver las demandas de cuidado y poder construir una trayectoria laboral en igualdad de posibilidades que sus pares varones. A ello se le suma la "naturalización" de la capacidad de las mujeres para cuidar que sigue siendo reforzada y adquiere nuevas aristas y manifestaciones que lejos están de un cuestionamiento a este orden estructurante de poder.

De este modo se invisibiliza la (sobre)carga que implican las responsabilidades asociadas al cuidado, y la restricción de la autonomía para cada mujer, sin embargo es absolutamente visible para reclamarle que estos pasos sean desempeñados con alta eficiencia, voluntad y también afectividad, lo que en muchos casos si así no se realizan se convierten en fuente de violencia hacia las mujeres, tanto en el ámbito de las relaciones de pareja, las intrafamiliares y las institucionales. Estas últimas abarcan desde el maltrato que, por ejemplo, recibe una mujer en el sector salud cuando acude a llevar a consulta a sus hijos e hijas y es acusada de desatenderlos, por lo cual llega muchas veces al extremo de la interrupción de un programa de transferencias condicionadas de ingresos por la inasistencia a esos controles, que paradójicamente muchas veces no es responsabilidad de la mujer sino del propio sistema por encontrarse desbordado de demandas.

Si se analiza desde el desempeño de su actividad laboral o productiva remunerada, la mujer que dedica tiempo a las actividades de cuidado, incluyendo su propio autocuidado, aunque sea mínimo, son tratadas severamente, no sólo por restar tiempo del trabajo productivo al reproductivo sino porque esta dedicación va a impactar en su trayectoria laboral y sin duda salarial, estableciendo un trato discriminatorio en relación con sus pares varones. A su vez, el impacto va a durar toda la vida ya que por ejemplo, la protección ante contingencias futuras

\footnotetext{
$\overline{8}$ El concepto de cuidado es multidimensional y abarca numerosos significados, implica la atención y satisfacción de aquellas necesidades físicas, biológicas, afectivas y emocionales que tienen las personas, sean o no dependientes, por estar transitando en los extremos de la vida (niñez, ancianidad) o por otras razones (enfermedades, discapacidad) requieren de una mayor cantidad de cuidados y/o de cuidados especiales. Estas acciones son reconocidas como trabajo, en su versión remunerada o no, e independientemente de su reconocimiento social (ELA: 2012).
} 
como la previsional va a quedar condicionada por ese uso del tiempo y de los mecanismos contributivos, ya que quienes más puedan demostrar horas trabajadas se benefician con más aportes, sistemas que a su vez no reconocen como trabajo a las labores de cuidado. En el caso de los ámbitos rurales, ésta es una necesidad aun más visible, dada la baja separación cotidiana de los espacios productivos de los reproductivos y el hecho de que el trabajo de cuidado es aun más demandante, vinculado a la nula disponibilidad de infraestructura y saneamiento básico, con factores ambientales que afectan la disponibilidad de tiempo ${ }^{9}$, así como de ausencia de oferta e infraestructura pública de cuidado, todo lo cual demanda por otra parte una transformación en la lógica de concentración del poder económico. Entre otras razones debido a que las formas de resolución son estratificadas y dependientes de los ingresos del hogar.

Lo anterior se correlaciona con los recorridos históricos de los regímenes de Bienestar, en los cuales la cuestión del cuidado fue considerada como responsabilidad principal de los hogares (y dentro de ellos, de las mujeres) y la participación del Estado reservada para aspectos muy específicos (por caso la educación escolar), o como complemento de los hogares allí cuando las situaciones así lo ameritan (por ejemplo, para el caso de hogares en situaciones de vulnerabilidad económica y social) terminó por moldear modelos de desestímulo al ingreso asalariado formal de las mujeres en el mercado de trabajo y por consiguiente las responsabilidades de cuidado para las mujeres.

Es decir, la problemática del cuidado va atravesando distintas situaciones y numerosos ejemplos construyen un mapa en el que las fronteras de la división sexual del trabajo se refuerzan cada día y siguen reproduciendo las asimetrías de poder en desmedro de la autonomía de las mujeres pero también de quienes requieren ser cuidados. A tal punto se refuerzan que quienes toman decisiones, a nivel gubernamental, sindical, empresarial o en los propios hogares, no sólo invisibilizan estas situaciones sino que habilitan estas fronteras con disposiciones, o con la ausencia de ellas, manteniendo un statu quo discriminatorio. De allí que se ha desarrollado una línea en relación con las fronteras del cuidado y la necesidad imperiosa de una agenda pública en tal dirección (Pautassi y Zibecchi, 2013).

En rigor y considerado que se han producido avances en el reconocimiento de la complejidad del trabajo de cuidado, especialmente en los momentos de escasez, se admite que es un trabajo duro, cansador, rutinario, pero que también gratifica, es fuente de afectos y de amor, y que actúa principalmente sobre la identidad de las mujeres y con efectos claros sobre los destinatarios. De ahí que aún se siga prestando, generación tras generación, de manera no remunerada, con compromiso y amor por la persona cuidada y con garantías de calidad, la que a su vez es difícil de medir y va a ser variable de acuerdo con los contextos y modelos sociales 
adoptados. Sin embargo, otra vez la frontera va a ser determinante cuando se trata del cuidado mercantilizado, en el que las exigencias aumentan y también se exige compromiso y calidad, pero nunca se repara en relación con los bajos salarios que en general reciben las trabajadoras en hogares particulares ${ }^{10}$. Es que ser mujer y cuidar, incluso más, adoptarlo como ocupación, habilita a la sociedad a interpretar que el altruismo debe regir sus elecciones y por lo tanto no se analiza que el hecho de ser mujer y realizar una tarea "devaluada" como es el cuidado opera como factor de discriminación salarial, que afecta a todas, pero es aun más severo en las tareas de cuidado remunerado (Folbre, 2001).

La reflexión sobre el cuidado ha motivado una profusa producción teórica y académica, en la que el aporte de la teoría feminista y del activismo del movimiento de mujeres ha sido notable y determinante para impulsar cambios en el plano político e institucional. Al respecto, uno de los abordajes que ha cobrado notoriedad fue la consideración del cuidado desde un enfoque de derechos humanos de modo de poder situar la titularidad del cuidado en la persona desvinculada de la condición o posición que ocupe. El reconocimiento del cuidado como derecho implica incorporar estándares y principios a la actuación de los Estados democráticos en las situaciones concretas, pero también contribuye a la consecución de políticas y prácticas equitativas, al mismo tiempo que aporta indicadores para la verificación de su cumplimiento, los cuales cobran una centralidad insoslayable para garantizar los derechos de cada persona que deba cuidar y a su vez pueda cuidarse, como también para quienes necesitan ser cuidados (Pautassi, 2007a).

Retomando las agendas globales, pero con impacto local, es necesario que la agenda post 2015 incluya las obligaciones derivadas del derecho al cuidado, en el entendido que las desigualdades en el mundo de las relaciones de cuidado suelen verse en muchos casos como una discriminación encubierta o simplemente no verse y asumirse como parte del nuevo escenario de un mundo "moderno" signado por la inserción masiva de las mujeres en el mercado de trabajo remunerado. Si bien una persona que cuida y la que recibe el cuidado no se encuentran en igualdad de condiciones, quienes deben ocuparse del cuidado ejercen estructuras asimétricas de unos sobre otros. $\mathrm{Y}$ así se sigue reproduciendo en forma interminable esta suerte de "cadena de la desigualdad". Por otra parte, a medida que las mujeres logran sortear las desigualdades y las discriminaciones en el mundo público, a partir de planes de igualdad de oportunidades, de los convenios internacionales igualitarios, de las acciones positivas, no se ha avanzado en considerar nuevas formas jurídicas de regular el cuidado, que trasciendan las regulaciones laborales para trabajadoras asalariadas (Pautassi, 2007a). Y a ello debe agregarse que se evidencian pocos cambios en la división sexual del trabajo al interior del hogar, donde los varones solo han avanzado, en el mejor de los casos, en asumir algunas actividades, pero

\footnotetext{
${ }^{10}$ Si de paradojas se trata, recién en el año 2013, con la sanción de la Ley 26844 que establece el Régimen Especial de Contrato de Trabajo para el Personal de Casas Particulares, el cual deroga el decreto 326 de 1956, y por primera vez se avanza en la equiparación -casi completa-de los derechos de las trabajadoras del resto de los asalariados formales. Recientemente dicha norma ha sido reglamentada y está a cargo de la Administración Nacional de Seguridad Social (ANSES) los beneficios de seguridad social incorporados.
} 
lejos se está en efectivizar la corresponsabilidad del cuidado ${ }^{11}$.

Concordantemente, estas asimetrías pasadas y presentes, remiten a cómo se puede incorporar en una lógica de derechos la complejidad del cuidado, de modo de desvincularlo de la condición de trabajador/a asalariado o con un "sujeto beneficiario" o "receptor" de una política de cuidado, sino cómo se puede reconocer como un principio inherente a la igualdad de oportunidades y de trato. La consideración del cuidado como derecho remite a la idea de derecho universal, independientemente del estado de necesidad que esté transitando la persona o de su inserción asalariada formal. A su vez, implica analizar el cuidado como obligación y las múltiples implicancias y derivaciones que trae aparejada, entre otras, si es una obligación privada o pública.

El derecho a cuidar, a ser cuidado y a cuidarse (autocuidado) tiene su correlato en la obligación de cuidar (Pautassi, 2007a). Implica, por lo tanto, un conjunto de obligaciones negativas, características de los derechos económicos sociales y culturales (DESC), como no entorpecer los servicios de centros infantiles, no impedir el acceso de un adulto mayor al sistema de salud; pero principalmente incluye obligaciones positivas, que se enrolan en proveer los medios para poder cuidar y derivar el cuidado, para garantizar que el cuidado se lleve adelante en condiciones de igualdad y sin discriminación y que no sólo se concedan para un grupo acotado de la población - por caso vinculado al empleo asalariado formal- sino que sean garantizados a todas las personas.

En concordancia, existen sujetos obligados a proveer el cuidado, desde los miembros de la pareja para con sus hijos e hijas, o de los hijos varones y mujeres para con sus progenitores en situación de autonomía relativa, pero también es el Estado, en determinados casos, quien también se encuentra obligado a "hacer". Esto es, no sólo el Estado debe no entorpecer que una madre amamante a su hijo o hija, sino que además le debe proveer las condiciones necesarias para ello. En el caso que trabaje en el ámbito productivo debe otorgarle licencia o un espacio físico para amamantar, tanto si es trabajadora del sector público o en el sector privado, como también debe otorgar licencias para los padres varones para que asuman conjuntamente la coresponsabilidad que les compete en materia de cuidado y desarrollo de cada niño, niña y adolescente o de sus padres ya adultos mayores. A su vez, la obligación positiva del Estado implica la imposición a terceros de ciertas y determinadas obligaciones, como en este caso, la obligatoriedad de los empleadores privados de que efectivamente provean la infraestructura de cuidado o de las licencias legalmente contempladas. En rigor, se trata de garantizar el derecho al cuidado, en tanto derecho universal y propio de cada persona, sea que deba cuidar o que necesite cuidados.

\footnotetext{
${ }^{11}$ Argentina no cuenta con una encuesta de uso del tiempo, pero a modo de ejemplo en el año 2005 se relevó información sobre uso del tiempo en la ciudad de Buenos Aires y mostró lo siguiente: la mayoría de las mujeres (92\%) participa en actividades de trabajo doméstico no remunerado para el propio hogar, mientras que varios varones también lo hacen, pero en una proporción menor (65,8\%). En relación con las actividades específicas de cuidado, las mujeres las realizan en un 30,9\%, y los varones en un $19,6 \%$ y en conjunto, y en promedio, las mujeres dedican a estas actividades el doble de tiempo que los varones (Esquivel et al, 2011). Estudios de caso posteriores mostraron resultados semejantes (ELA; 2012).
} 


\section{LAS APROXIMACIONES AL CUIDADO ${ }^{12}$}

En América Latina "se cuida como se puede, se es cuidado también como y cuando se puede”, lo cual perdura como una metáfora muy potente que, fundamentada en evidencia empírica disponible en la región, da cuenta no sólo del déficit de atención sino también del impacto que tiene sobre las personas, en especial en las mujeres. Cabe recordar que existe una acentuada carencia en la producción de información en relación no sólo con las demandas de cuidado: son pocos los países que han incorporado encuestas de uso del tiempo u otras herramientas que permitan indagar sobre la división sexual del trabajo y el análisis de las desigualdades y discriminaciones presentes no puede - ni debe- circunscribirse a un examen de indicadores en el mercado de trabajo remunerado. Han sido muchas voces que llevan décadas alertando sobre la parcialidad de este enfoque y su escasa utilidad para diseñar políticas efectivas correctivas y transformadoras de las situaciones vigentes (entre otras, Picchio, 2001; ONU-Mujeres, 2012; Marco y Rico, 2013, Razavi, 2007; Tronto, 2006).

Concordantemente, es necesario que todo análisis que se realice requiera la consideración de múltiples dimensiones que incluyen: i) el uso y la distribución del tiempo total de trabajo y las responsabilidades de cuidado, y i) las desigualdades en la inserción en el mercado de trabajo remunerado, tanto en la posibilidad de acceso en igualdad de condiciones como en relación al análisis de las trayectorias laborales y los diferenciales en la calidad de la ocupación. Sin embargo, los sistemas estadísticos no permiten estos abordajes, y con cierto grado de resistencia van incorporando lentamente algunos de los indicadores de género necesarios para realizar estos análisis. Específicamente con el cuidado, la debilidad del sistema de información en cada uno de los países configura un claro ejemplo de la falta de presencia que este tema ha tenido en la agenda política, dando cuenta de una expresa omisión en su tratamiento.

Otro de los aspectos centrales que circunscribe las formas de resolución del cuidado se relaciona con las condiciones de inserción en el mercado laboral, esto es las desigualdades existentes entre las trabajadoras asalariadas formales con empleo registrado y quienes desempeñan tareas por cuenta propia o en el sector informal, aplicándose un criterio de selectividad estricto. De este modo, las primeras gozan de protección contra el despido discriminatorio -en razón de matrimonio o embarazo, cuentan con licencias o permisos para el nacimiento de sus hijos e hijas que, salvo disposiciones y regímenes locales que han ampliado las mismas, por Ley de Contrato de Trabajo (LCT) es de 90 días de licencia remunerada, la que no es pagada por el empleador sino por el sistema de seguridad social. Asimismo durante el primer año de vida cuentan además con permisos de lactancia que varían en los distintos países de la región, dejando el resto del ciclo vital de crecimiento del niño o de la niña con escasos permisos para su atención. El sesgo de género en el tratamiento legislativo es claro al considerar permisos su-

12 Sigo aquí los argumentos principales desarrollados en Pautassi (2013). 
mamente restrictivos para los padres por nacimiento del hijo o hija: dos días hábiles por LCT (Pautassi y Rico, 2011) ${ }^{13}$. Por otra parte, persisten los sesgos al establecer la obligatoriedad de la guarderías -sea su provisión directa o monto equivalente- a partir de la presencia de 50 trabajadoras mujeres, presuponiendo la norma que quienes tienen dificultades para conciliar las esferas productivas con las reproductivas son solamente las mujeres.

Tradicionalmente se han considerado montos en conceptos de asignaciones familiares para asalariados registrados, en concepto de cobertura de contingencias vinculadas con el crecimiento y expansión del núcleo familiar: matrimonio, nacimiento, adopción, escolaridad de los hijos e hijas, que en general el perceptor tradicional del mismo ha sido el varón, ya que las mujeres han tenido menor inserción asalariada formal.

Los sesgos de género continúan con la falta de regulaciones para asumir el cuidado de personas adultas mayores, personas enfermas o con discapacidad, que están ausentes de las legislaciones laborales en América Latina en general y en Argentina en particular. Sólo con esta mirada sobre algunos de los datos disponibles se puede constatar que las formas de inserción laboral remunerada de varones y mujeres, con escasos niveles protectorios, dejan poco margen para asumir las tareas de cuidado de las personas dependientes del núcleo del hogar. Pero, además, dejan menos margen para poder dedicarse al autocuidado, el que incluye desde la asistencia y los controles de salud mínimos hasta el ejercicio del derecho al tiempo libre dedicado a descanso, recreación, actividades culturales.

En otros términos, la asignación cultural, social y económica de las responsabilidades de cuidado en las mujeres impregna las políticas económicas y sociales de la región, impactando sobre el déficit en la provisión de infraestructura social de manera diferencial en varones y en mujeres, a lo cual se suma la desigualdad protectoria de los regímenes laborales, la debilidad de la inversión en todas las áreas que demandan cuidado y que en consecuencia no sólo impacta en las condiciones de vida sino que supone una mayor carga de trabajo y de gasto en los hogares para realizar algunas de las tareas de cuidado, y por lo tanto condicionan la cantidad de horas disponibles para el trabajo remunerado. A su vez, y debido al alto nivel de estratificación que estructura al cuidado, quienes menos tienen verán reducidas sus posibilidades de acceder a servicios de cuidado por la necesidad de adquirirlos en el mercado a costos que no pueden solventar. Esta situación actúa en desmedro del tiempo y de las oportunidades de las mujeres, pero también recae directamente sobre quienes requieren de cuidado, como las personas con discapacidad, enfermos o personas adultas mayores, y en el caso de niños, niñas y adolescentes, limita el desarrollo de una vida plena en hogares con menores niveles de ingreso. En otros términos, las situaciones de desigualdad persistentes en la región afectan a todos y a todas, la diferencia es la intensidad, recargando más a las mujeres y no a todas por igual, lo que unido 
a la debilidad de los servicios de cuidado a nivel institucional implica que las mujeres deben desplegar múltiples estrategias, desde la inserción laboral hasta las de cuidado familiar, que limitan su tiempo, condicionan el tipo de elección que realizan, impactando en la situación socioeconómica de los hogares y centralmente en el ejercicio de su autonomía.

Estas, entre otras tensiones, dan cuenta no sólo de la urgencia por solucionar la situación de las mujeres, especialmente como prestadoras genéricamente asignadas, pero también la de los destinatarios del cuidado, mas la solución no debe ser a partir de respuestas aisladas como las que se han venido produciendo paulatinamente en muchos países sino con una respuesta integral y transversal, transformadora de las asimetrías y discriminaciones existentes. Y allí los derechos humanos actúan como un marco de referencia ineludible para poder comenzar el proceso de transformación.

\section{EL CUIDADO DESDE UN ENFOQUE DE DERECHOS}

"Integrar derechos, transformar las políticas públicas" es una de las máximas que el nuevo milenio ha promovido a nivel internacional pero particularmente regional, actuando no sólo como expresión de deseos sino como una metodología de aplicación en los contextos gubernamentales locales.

El desembarco del cuidado como derecho se produjo en el marco de la X Conferencia Regional de la Mujer de América Latina y el Caribe, celebrada en Quito, Ecuador, en 2007, tras la cual los gobiernos firmaron el Consenso de Quito donde se comprometieron a adoptar medidas para atender la reproducción social, el cuidado y el bienestar de la población entendidos todos como responsabilidades públicas. Así, el Consenso de Quito asume como compromiso "formulary aplicar políticas de Estado que favorezcan la responsabilidad compartida equitativamente entre mujeres y hombres en el ámbito familiar, superando los estereotipos de género, reconociendo la importancia del cuidado y del trabajo doméstico para la reproducción económica y el bienestar de la sociedad como una de las formas de superar la división sexual del trabajo". Asimismo se acuerda "adoptar medidas en todas las esferas de la vida particular, en los ámbitos económico y social, incluidas reformas institucionales, para garantizar el reconocimiento y el aporte al bienestar de las familias y al desarrollo promover su inclusión en las cuentas nacionales”.

Estos acuerdos fueron retomados nuevamente en el Consenso de Brasilia (2010) durante la XI Conferencia Regional de la Mujer de América Latina y el Caribe, donde los Estados, "reconociendo que el acceso a la justicia es fundamental para garantizar el carácter indivisible e integral de los derechos humanos, incluido el derecho al cuidado, señalando que el derecho al cuidado es universal y requiere medidas sólidas para lograr su efectiva materialización y la corresponsabilidad por parte de toda la sociedad, el Estado y el sector privado para comprometerse, entre otros puntos: [...] a) adoptar todas las medidas de política social y económica 
necesarias para avanzar en la valorización social y el reconocimiento del valor económico del trabajo no remunerado prestado por las mujeres en la esfera doméstica y del cuidado; b) fomentar el desarrollo y el fortalecimiento de políticas y servicios universales de cuidado, basados en el reconocimiento del derecho al cuidado para todas las personas y en la noción de prestación compartida entre el Estado, el sector privado, la sociedad civil y los hogares, así como entre hombres y mujeres, y fortalecer el diálogo y la coordinación entre todas las partes involucradas; c) adoptar políticas que permitan establecer o ampliar las licencias parentales, así como otros permisos de cuidado de los hijos e hijas, a fin de contribuir a la distribución de las tareas de cuidado entre hombres y mujeres, incluidos permisos de paternidad irrenunciables e intransferibles, que permitan avanzar en la corresponsabilidad" $\left(\operatorname{art.} 1^{0}\right)$.

Recientemente, el Consenso de Santo Domingo, aprobado en el marco de la duodécima Conferencia Regional de la mujer de América Latina y el Caribe, de octubre de 2013, establece que los Estados se comprometen a: "Promover la mejora del acceso de las mujeres al empleo decente, redistribuyendo las tareas de cuidado entre Estado, mercado y sociedad y entre hombres y mujeres, y facilitando la capacitación y uso de la tecnología, el autoempleo y la creación de empresas en el sector científico-tecnológico, así como aumentar la proporción de mujeres que ocupan puestos en áreas en que se encuentran infrarrepresentadas, como los ámbitos académico, científico, tecnológico y de las tecnologías de la información y las comunicaciones”(art. 37).

Luego establece en el art. 57: "Reconocer el cuidado como un derecho de las personas $y$, por lo tanto, como una responsabilidad que debe ser compartida por hombres y mujeres de todos los sectores de la sociedad, las familias, las empresas privadas y el Estado, adoptando medidas, políticas y programas de cuidado y de promoción de la corresponsabilidad entre mujeres y hombres en la vida familiar, laboral y social que liberen tiempo para que las mujeres puedan incorporarse al empleo, al estudio y a la política y disfrutar plenamente de su autonomía”. Es decir, ya se cuenta con instrumentos donde se incluye la consideración del cuidado como un derecho y por lo tanto es exigible.

En rigor, el enfoque de derechos explicita un reconocimiento de la relación directa existente entre el derecho, el empoderamiento de sus titulares, la obligación correlativa y la garantía, todo lo cual conjuga en una potencialidad que puede actuar como una forma de restablecer equilibrios en el marco de situaciones sociales marcadamente desiguales (Pautassi, 2007a).

Concordantemente, el avance desde el reconocimiento a la satisfacción plena generalmente es paulatino y el Estado está jurídicamente obligado a garantizar el contenido mínimo de los derechos incorporados y no puede escudarse en la falta de recursos disponibles para justificar su accionar si éste induce a un sector de la población por debajo del estándar mínimo de protección de ese derecho. En rigor, el enunciado de metas de cumplimiento por parte del Estado no cubre su obligación positiva de satisfacer por completo cada uno de los derechos reconocidos en los pactos y en las Constituciones o leyes internas de los países, sino que debe efectivamente 
alcanzarlo. Tal como fue señalado, debido a que los derechos humanos son universales, indivisibles e interdependientes (art. 5 Plataforma de Acción Conferencia de Derechos Humanos de Viena, 1993); el contenido mínimo, la utilización del máximo de recursos disponibles en forma progresiva y con una clara prohibición de regresividad y basado en garantías de igualdad y no discriminación, acceso a la justicia y acceso a la información pública, conforman estándares que actúan todos en forma transversal al conjunto de la política económica, social y cultural.

A su vez, y siguiendo la argumentación principal de este enfoque, el empoderamiento de las y los destinatarios de políticas públicas es uno de los principales efectos, el que comienza por reconocer que los mismos son titulares de derechos que generan obligaciones al Estado y no meros "beneficiarios" de políticas estatales. El enfoque, en tanto metodología teórica operativa que persigue un objetivo político que es la equidad social, étnica y de género, busca revertir la concepción de un destinatario pasivo del accionar del Estado, para precisamente diseñar políticas e instrumentos de intervención que permitan un ejercicio efectivo de los derechos económicos, sociales y culturales (DESC) y del conjunto de derechos humanos.

Y allí obtiene una centralidad mayúscula que busca desafiar la relación pasiva que existe entre el sujeto titular de derechos y la discrecionalidad de la administración pública para garantizarlos o, en términos de la relación del cuidado, busca romper la lógica binaria de actividad/pasividad entre el proveedor o dador de cuidado y el destinatario, que no sólo incluye la práctica interpersonal de cuidar al otro sino que demanda un conjunto integrado de acciones transversales al respecto.

Aplicar el enfoque de derechos al cuidado significa transformar la lógica actual de tratamiento del cuidado para pasar a considerar que cada sujeto autónomo, portador de derechos, puede y debe exigir la satisfacción de sus demandas de cuidado, independientemente de su situación de vulnerabilidad o dependencia, ya que el deber de provisión de cuidados que el derecho le confiere no se asienta en su necesidad sino en su condición de persona. Es decir, no debe argumentar que necesita cuidado porque es un niño o niña o un enfermo cuya situación lo requiere sino que el Estado y demás sujetos obligados -como ambos progenitores en relación con sus hijos e hijas- deben brindárselo independientemente de su situación, sólo por ser persona.

En rigor, el ejercicio de la autonomía es resituado en toda su amplitud, en tanto el sujeto titular del derecho al cuidado puede exigir y decidir las opciones en torno al cuidado de manera independiente, aun cuando se necesite de la asistencia y el apoyo de los demás para lograrla. Independizar la necesidad de cuidado de su exigibilidad es el principal aporte del enfoque de derechos.

Sin embargo, con el reconocimiento del derecho al cuidado no se culmina con las demandas del mismo sino que a partir de este reconocimiento comienza a actuar la metodología incorporada en el enfoque de derechos para transformar la dinámica actual y que se efectivicen las garantías que este derecho incluye. Y esta premisa es aplicable al conjunto de DESC y a la estatalidad toda, especialmente a las políticas públicas y sociales. 
En síntesis, si bien los tratados y pactos internacionales de derechos humanos no han incluido explícitamente "el derecho a cuidar, a ser cuidado y a cuidar(se)", sin embargo han incorporado derechos sociales como el derecho a una alimentación adecuada o a la seguridad social en su dimensión más amplia que incluye a toda la población y no únicamente a los asalariados formales, el derecho a la salud, a la educación, a la cultura. A su vez, la consideración como derecho universal al cuidado abre el espectro de incorporación de los adultos mayores como receptores de cuidado, que en general no están reconocidos en los ordenamientos laborales como sujetos de cuidado por parte de los activos. Sólo niños, niñas y adolescentes entran en el ámbito de responsabilidad de las trabajadoras mujeres (en el marco de las regulaciones laborales), pero los adultos mayores están rara vez mencionados. Estos sesgos de género contenidos en las regulaciones laborales y en la dinámica de los mercados de trabajo van a tener un impacto diferenciado según la existencia de sistemas de políticas sociales universales que permitirán amortiguar ciertos efectos, que si el ingreso de las mujeres al mercado de trabajo se hace en el ámbito de la informalidad y del desarrollo de precarios sistemas de protección social.

De ahí que el énfasis queda puesto en el reconocimiento explícito del cuidado como derecho universal, indisponible y no sujeto a concesiones para grupos especiales, sino que se garantice para todos los habitantes. La advertencia que debo formular es que en el afán de hacer visible el trabajo que significa el cuidado para las mujeres, además de las responsabilidades que implica, no se reafirme su responsabilidad en términos jurídicos sino que sea compartida con los varones y a nivel de toda la sociedad.

La consideración del derecho al cuidado universal es un primer paso para distribuir las responsabilidades de cuidado en todos y cada uno de los integrantes de la sociedad y no sólo en las mujeres, ya que precisamente estamos reconociendo que es un derecho de todas y todos que nos incluye, nos protege, nos empodera y también nos obliga.

\section{LA AGENDA EMERGENTE Y URGENTE}

Si bien son altamente destacables los avances en el reconocimiento del derecho al cuidado que se han producido en los últimos años en la región, aun resultan débiles las medidas concretas, quedando en muchos casos como enunciados retóricos.

Por lo tanto, resulta indispensable cuestionar la división sexual del trabajo y buscar la mejor manera de redistribuir las obligaciones de cuidar, en el reconocimiento de los derechos de quienes necesitan ser cuidados y de quienes de un modo u otro han de proporcionárselos. Una mejor visualización, tanto del cuidado como de otras actividades humanas beneficiosas para la sociedad -pero invisibles según la actual concepción de la realidad económica- es una de las premisas más importantes para abordar en la actualidad. La consideración del cuidado como un derecho universal que incluya a todos y a todas, en su potestad de reclamar el derecho 
a ser cuidado, a cuidar y a cuidarse (autocuidado), significa que no se trata sólo de impulsar acciones que aumentan la oferta de servicios reproductivos, indispensables por cierto, sino que transversalmente se aborden las responsabilidades vinculadas en sentido amplio, de modo de romper con el sistema estratificado que se ha desarrollado en torno al cuidado, ya que resulta altamente dependiente de los recursos con los que cuenta cada persona y las familias.

Nuevamente, se pueden identificar numerosos niveles que se deben tener en consideración a la hora de evaluar los esfuerzos y las políticas implementadas por los Estados en relación con los compromisos asumidos en los pactos y tratados internacionales. En rigor, el desarrollo de indicadores de derechos humanos es una de las estrategias que, integrada a otros elementos de política pública, especialmente la transversalización del enfoque de derechos, género y etnia, permite aproximar vías de evaluación y de diseño de políticas públicas que avancen en la efectivización del derecho al cuidado. La utilización de indicadores de derechos humanos como un mecanismo de exigibilidad presenta múltiples oportunidades, tanto para los sujetos portadores del derecho, principalmente porque los empodera en la titularidad del derecho y en su vínculo con el Estado como responsable de satisfacer el contenido de los mismos. A su vez, les ofrece a los Estados la posibilidad de evaluar las políticas implementadas, permitiendo realizar las transformaciones necesarias. Hasta tanto no se asimile que la organización social del cuidado es determinante para el desarrollo económico, social, político, para el empleo, para las políticas sociales, empresarias, poco lograremos avanzar en modelos sustentables y seguiremos reproduciendo las asimetrías señaladas. Por lo mismo, si esta organización social del cuidado es injusta, inexorablemente se reflejará en las respuestas estatales.

Por otra parte, el empoderamiento también promueve nuevas instancias de negociación de las responsabilidades en el interior de la pareja y de las relaciones familiares, y posibilita a las mujeres ejercer su autonomía con relación al destino de "cuidadoras" que les ha asignado el modelo patriarcal, que a la vez es reforzado por la burocracia asistencial y los modelos económicos imperantes. Los aportes de la teoría y economía feminista son un elemento de quiebre fundamental contra falsos argumentos en torno a la "funcionalidad" de lo público y lo privado. Del mismo modo, las impugnaciones al supuesto carácter igualitario de la ciudadanía complementan la base necesaria para no descansar en el proceso de "cambio cultural" las soluciones a las discriminaciones señaladas sino adoptar políticas públicas activas dirigidas a sujetos autónomos y titulares de derechos. Sin embargo, el proceso de transformación cultural -y no simple cambio- no debe minimizarse ya que es uno de los enclaves patriarcales más severos de desmontar.

Sólo en la medida en que se integre al conjunto del accionar estatal se avanzará en una mayor institucionalidad y garantías en materia de derechos humanos e iremos construyendo una agenda post 2015 más igualitaria. Resta trabajar fuertemente sobre la división sexual del trabajo dentro de las relaciones de pareja y de las relaciones sociales de género. Se trata no sólo de procurar mejores condiciones para que las mujeres presten cuidado sino precisamente 
de que el cuidado sea un derecho de cada persona, prestado bajo parámetros de calidad y en cantidad suficientes y no exclusivamente por mujeres.

Bajo estos mínimos irrenunciables la agenda pública 2015 se construye en base a obligaciones a cumplir y no en base a retos o desafíos. Este es el punto, que a mi modo de ver, va a transformar la agenda de desarrollo y la institucionalidad pública en América Latina. Adoptado este principio, se requieren diseños universales e interdependientes que implementan el derecho al cuidado de cada persona.

\section{REFERENCIAS BIBLIOGRÁFICAS}

Abramovich Víctor y Laura Pautassi (2009), "El enfoque de derechos y la institucionalidad de las políticas sociales”, en Víctor Abramovich y Laura Pautassi (comps.), La revisión judicial de las políticas sociales. Estudio de casos, Buenos Aires, Del Puerto.

ELA (2012): "De eso no se habla: el cuidado en la agenda pública". Estudio de Opinión sobre la organización del Cuidado, Equipo Latinoamericano de Justicia y Género, Buenos Aires, enero de 2012 (disponible en: www.ela.org.ar).

Esquivel, V., Faur, E. y E. Jelin (2012) Las lógicas del cuidado infantil. Entre las familias, el Estado y el mercado. Buenos Aires: IDES - UNFPA- UNICEF.

Folbre, Nancy (2001), The Invisible Heart. Economics and Family Values, Nueva York, The New York Press.

Marco, Flavia y Rico, María Nieves (2013) Cuidado y políticas públicas: debates y estado de situación a nivel regional. En: Laura Pautassi y Carla Zibecchi (coordinadoras) Las fronteras del cuidado. Agenda, derechos e infraestructura" Buenos Aires, Editorial Biblos, 2013, págs.: 27-58

OEA (Organización de Estados Americanos) (2011), Indicadores de progreso para medición de derechos contemplados en el Protocolo de San Salvador (oea/Ser. 1/xxv.2.1.gt/pssi/ doc.2/11), Grupo de Trabajo Protocolo para el análisis de los informes previstos en el Protocolo de San Salvador, Washington, diciembre.

Pautassi, Laura (2007a) El cuidado como cuestión social desde el enfoque de derechos. Serie Mujer y Desarrollo No ${ }^{\circ}$, Santiago de Chile, CEPAL. 
Pautassi, Laura (2007b) “iCuánto Trabajo mujer! El género y las relaciones laborales”. Buenos Aires, Capital Intelectual.

Pautassi, L (2013). Perspectivas actuales en torno al enfoque de derecho y cuidados: autonomía en tensión. En: Pautáis, L. y Zibecchi, C. (comp.) Las Fronteras del Cuidado, Agenda, derechos e infraestructura, Buenos Aires, Biblos.

Pautassi, L. (2014) (directora) "Marginaciones sociales en el área metropolitana de Buenos Aires. Acceso a la justicia, capacidades estatales y movilización legal”. Buenos Aires, Editorial Biblos, en prensa.

Pautassi, Laura y María Nieves Rico (2011), "Licencias para el cuidado infantil. Derecho de hijos, padres y madres", Desafios. Boletín de la infancia y adolescencia sobre el avance de los Objetivos de Desarrollo del Milenio, № 12, Santiago de Chile: CEPAL-UNICEF.

Pautassi, L. y Gamallo, G. (2012) (directores) “¿Más derechos, menos marginaciones? Políticas sociales y bienestar en Argentina” Buenos Aires: Biblos, 2012

Pautassi, Laura y Zibecchi, Carla (coords) (2013) Las fronteras del cuidado. Agenda, derechos e infraestructura. Buenos Aires: ELA-Editorial Biblos.

Picchio, Antonella (2001), "Un enfoque macroeconómico ampliado de las condiciones de vida”, conferencia inaugural de las jornadas “Tiempos, trabajos y género”, Universidad de Barcelona.

Razavi S (2007). The Political and Social Economy of Care in a Development Context Conceptual Issues, Research Questions and Policy Options. UNRISD www.unrisd.org/80256B3Co05BCCF9/ (httpAuxPages)/2DBE6A93350A7783C12573240036D5Ao/\$file/Razavi-paper.pdf

Rico, María Nieves (2011), “Crisis del cuidado y políticas públicas: el momento es ahora”, Las familias latinoamericanas interrogadas. Hacia la articulación del diagnóstico, la legislación y las políticas, Serie Seminarios y Conferencias, No 61 (LC/L.3296-P), Santiago de Chile, Comisión Económica para América Latina y el Caribe (CEPAL), Febrero.

Rodríguez Enríquez, C. y Pautassi, L. (coord.) (2014) La organización social del cuidado de niños y niñas. Elementos para la construcción de una agenda de cuidados en Argentina. Buenos Aires, ELA; CIEPP y ADC. 
Rodríguez Enríquez, C. (2007) Empleo femenino, economía del cuidado y políticas públicas. Algunas consideraciones desde la experiencia argentina”. En: M. A. Carbonero Raimundí y S. Levín (comps) Entre familia y trabajo. Relaciones, conflictos y políticas de género en Europa y América Latina. Rosario: Editorial Homo Sapiens. 2007.

Tronto, Joan (2006), "Vicious circle of privatized caring", Socializing Care: Feminist Ethics and Public Issues, en Maurice Hamington y Dorothy Miller (eds.), Socializing Care: Feminist Ethics and Public Issues, Lanham, Rowman and Littlefield.

\section{CURRÍCULUM VITAE}

\section{Laura C. Pautassi}

Abogada, Doctora en Derecho, Área Derecho Social (Universidad de Buenos Aires. Investigadora Independiente del CONICET. Docente e investigadora permanente del Instituto de Investigaciones Jurídicas y Sociales “Ambrosio L. Gioja”, Facultad de Derecho (UBA). Directora del Grupo de Trabajo Interdisciplinario "Derechos Sociales y Políticas Públicas” (www.dspp. com.ar) y del Proyecto Interdisciplinario de Investigación UBACYT 2012 - 2014 "Derechos sociales, provisión de bienestar y marginación social en Argentina”.

laurapautassi@derecho.uba.ar 